\title{
Erratum to: Maternal intake of seafood and supplementary long chain n-3 poly- unsaturated fatty acids and preterm delivery
}

Anne Lise Brantsæter ${ }^{1 *}$, Linda Englund-Ögge ${ }^{2}$, Margareta Haugen ${ }^{1}$, Bryndis Eva Birgisdottir ${ }^{3}$, Helle Katrine Knutsen ${ }^{1}$, Verena Sengpiel ${ }^{2}$, Ronny Myhre ${ }^{4}$, Jan Alexander ${ }^{5}$, Roy M. Nilsen ${ }^{6}$, Bo Jacobsson ${ }^{4,7}$ and Helle Margrete Meltzer ${ }^{1}$

\section{Erratum}

In the original publication of this article [1], the categories for lean and fatty fish in Fig. 2 should have been listed as servings per week and not per day. Please see updated figure below.

\begin{abstract}
Author details
'Department of Environmental Exposure and Epidemiology, Domain of Infection Control and Environmental Health, Norwegian Institute of Public Health, P.O. Box 4404NydalenNO-0403 Oslo, Norway. ${ }^{2}$ Department of Obstetrics and Gynecology, Sahlgrenska University Hospital, Gothenburg, Sweden. ${ }^{3}$ Unit for Nutrition Research, Landspitali University Hospital and University of Iceland, Reykjavik, Iceland. ${ }^{4}$ Department of Genetics and Bioinformatics, Domain of Health Data and Digitalisation, Norwegian Institute of Public Health, Oslo, Norway. ${ }^{5}$ Office of the Director-General, Norwegian Institute of Public Health, Oslo, Norway. ${ }^{6}$ Department of Health and Social Sciences, Bergen University College, Bergen, Norway. ${ }^{7}$ Department of Obstetrics and Gynecology, Sahlgrenska Academy, Gothenburg University, Gothenburg, Sweden.
\end{abstract}

Received: 25 January 2017 Accepted: 31 January 2017

Published online: 10 February 2017

\section{Reference}

1. Brantsæter AL, et al. Maternal intake of seafood and supplementary long

chain $n-3$ poly-unsaturated fatty acids and preterm delivery. BMC Pregnancy Childbirth. 2017;17:41. doi:10.1186/s12884-017-1225-8.

\footnotetext{
* Correspondence: AnneLise.Brantsaeter@fhi.no

${ }^{1}$ Department of Environmental Exposure and Epidemiology, Domain of Infection Control and Environmental Health, Norwegian Institute of Public Health, P.O. Box 4404NydalenNO-0403 Oslo, Norway
} 


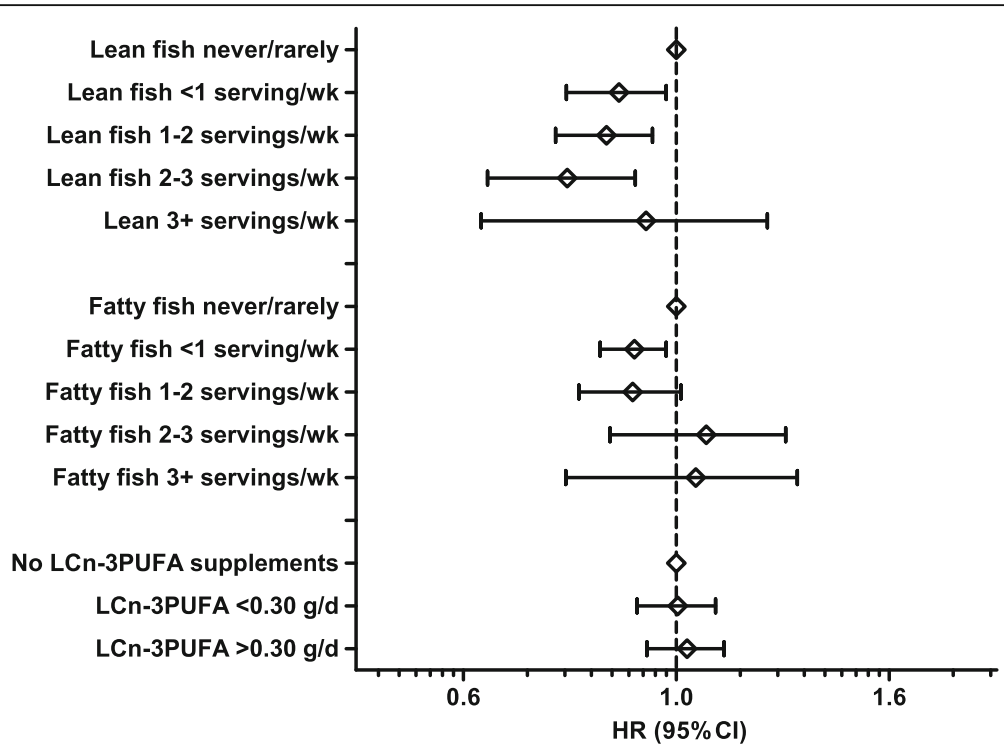

Fig. 2 Associations (hazard ratio (HR) and 95\% confidence intervals (CI)) between intakes of lean fish, fatty fish and marine long chain n-3 polyunsaturated fatty acids (LCn-3PUFA) from supplements and preterm delivery. Intakes are mutually adjusted and adjusted for maternal age, pre-pregnancy BMI, height, parity, energy intake, maternal education, smoking, marital status, household income and previous preterm delivery. $N=67,007$ women in the Norwegian Mother and Child Cohort Study (MoBa) 2002-2008 